\title{
Byung-Chul Hans Diagnosen der Gegenwartsgesellschaft
}

\author{
Sammelbesprechung
}

Besprochene Bücher:

- Byung-Chul HAN, Digitale Rationalität und das Ende des kommunikativen Handelns, Berlin, Matthes E Seitz, 2013, 46 S., $17 €$

- Ders., Transparenzgesellschaft, Berlin, Matthes E Seitz, 2012, 92 S., $10 €$

- Ders., Agonie des Eros, Berlin, Matthes E Seitz, 2012, 76 S., $10 €$

- Ders., Müdigkeitsgesellschaft, Berlin, Matthes E Seitz, 2010, 72 S., $10 €$

Intensiviert das Internet die Demokratie? Oder untergräbt es die Fundamente der Politik? Bekanntlich liefert das Web unendlich viele Informationen natürlich nicht nur den Bürgern, sondern über die Bürger, werden längst überall individuelle Profile erstellt, die noch die geheimsten unbewussten Wünsche bei Kaufentscheidungen ans Tageslicht zerren, die die Werbung zu nutzen sucht.

Für Byung-Chul Han - koreanischer Herkunft und Professor für Philosophie an der Universität der Künste in Berlin - erscheint daher eine digitale Biopolitik nicht fern, die sich auf eine Transparenzgesellschaft stützen kann. Solche Wortgebilde liebt Han besonders und erfindet fleißig ähnliche: Müdigkeitsgesellschaft, Positivgesellschaft, Ausstellungsgesellschaft, Evidenzgesellschaft.

Damit, dass für ihn die Transparenzgesellschaft biopolitische Züge annimmt, greift Han einen Begriff Michel Foucaults auf, der allerdings Biopolitik erheblich struktureller auf eine umfassende Regierungstätigkeit des Staates bezieht, dessen Gegenstand das Leben selbst ist und gerade nicht ein Unbewusstes. Jedenfalls besitzt die Transparenzgesellschaft dadurch einen totalitären Zug der Gleichschaltung und nicht der Demokratisierung. In ihr wird nämlich alles nur positiv, nicht negativ betrachtet, vielmehr alles ökonomisch gleichgesetzt. »Das Geld «, schreibt Han, »das alles mit allem vergleichbar macht, schafft jede Inkommensurabilität, jede Singularität der Dinge ab. Die Transparenzgesellschaft ist eine Hölle des Gleichen.«(6) Aber gehört die Gleichheit nicht zur Demokratie?

Die Seele - ein merkwürdiger Begriff in solchen Zusammenhängen; indes hat Han auch Theologie studiert - bedarf dagegen des Geheimnisses, der Intransparenz, ohne von den anderen so ohne weiteres erblickt werden zu können. Aber wird nicht - muss man Han entgegnen - die Seele von Gott durchschaut, könnten wir uns gemäß der christlichen Eschatologie mit dem Internet auf dem Weg zur totalen Transparenzgesellschaft nach dem jüngsten Gericht befinden? 
Doch die Transparenzgesellschaft - so Han - beruht gerade nicht auf Wahrheit, die Repräsentation und Referenz, also Vermitteltheit braucht, gerade nicht die Unmittelbarkeit der Präsenz und des Mausklicks. Damit rekurriert Han freilich auf ein prämodernes Wahrheitsverständnis, das spätestens seit Nietzsche und Heidegger seine Unwahrheiten nicht mehr zu verbergen vermag.

Jedenfalls gerät die Transparenzgesellschaft für Han zu einer Ausstellungsgesellschaft, die ihren pornographischen Charakter enthüllt. Denn es geht nur noch um die Äußerlichkeit. Lust und Verführung gehören nicht dazu, da der Verführer nicht mit der Transparenz, sondern mit dem Schein arbeitet. Natürliche Nacktheit hat eine semantische Unschärfe, während der pornographischen der erotische Glanz fehlt und sie obszön erscheint, weil ihr jedes Geheimnis abgeht. Ihr fehlt die Innerlichkeit.

Derart kommt es gar zu einer Agonie des Eros. Han schreibt: »Die Liebe positivisiert sich heute zur Sexualität, die ebenfalls dem Leistungsdiktat unterworfen ist. Sex ist Leistung. Und Sexyness ist Kapital, das es zu vermehren gilt. Der Körper mit seinem Ausstellungswert gleicht einer Ware. Der Andere wird zum Erregungsobjekt sexualisiert. Man kann den Anderen, dem die Andersheit genommen worden ist, nicht lieben, sondern nur konsumieren. Er ist insofern auch keine Person mehr, als er zu sexuellen Teilobjekten fragmentarisiert wird. Es gibt keine sexuelle Persönlichkeit.« (19) Aus einer leistungsorientierten Gesellschaft eines enthemmten Neoliberalismus soll jeder Eros entwichen sein. Aber gibt es Erotik ohne Erregung, ohne Sex? Und gehört zur Liebe nicht nur Erotik, sondern auch Sexualität? Hans Eros scheint der christlichen Liebe, der Agape, der Nächstenliebe näher zu stehen, bedauert er doch ein Verschwinden der Seele.

In der Transparenzgesellschaft wird alles enthüllt und alles kontrolliert. Das schafft die Andersheit des Anderen ab. Nichts darf es geben, was man nicht erfasst. Der Andere wird daher auf den Exoten reduziert, dem man mit Neugier begegnet. In der globalisierten Welt gibt es nur noch Differenzen unter Gleichen. Es fehlen aber gerade die Grenzen, die Gräben, die Mauern, die aus dem Fremden, Exotischen einen derart Anderen machen, dass man vor ihm zurückschreckt, der eine immunologische Reaktion auslöst, weil man ihn als Feind begreifen muss.

So besitzt die Transparenzgesellschaft letztlich nichts Verbindendes mehr, erklärt sie niemanden mehr zum Feind, was zusammenschweißen würde. Damit nähert sich Han der Freund-Feind-Unterscheidung von Carl Schmitt an, dem katholischen Chefjuristen der Nazis. Derart verbreitet sich Bindungsarmut, Atomisierung und Fragmentierung, verblassen die traditionellen ethischen Normen. »Die Transparenzgesellschaft ist eine Gesellschaft des Misstrauens und des Verdachts, «so Han, »die aufgrund des schwindenden Vertrauens auf Kontrolle setzt. Die lautstarke Forderung nach Transparenz weist gerade darauf hin, dass das moralische Fundament der Gesellschaft brüchig geworden ist, dass moralische Werte wie Ehrlichkeit oder Aufrichtigkeit immer mehr an Bedeutung verlieren. An die Stelle der wegbrechenden moralischen Instanz tritt die Transparenz als neuer gesellschaftlicher Imperativ.« (79)

Dem muss man allerdings entgegnen, dass sich das mittelalterliche Christentum lieber auf den blinden Gehorsam und die Beichte stützte, als der Moral der Gläubigen zu ver- 
trauen. Erst die Aufklärer im 18. Jahrhundert hofften auf die Moral, während sich die militarisierte Gesellschaft des 19. lieber der Disziplin bediente.

Allemal kann dieses Lamento vom Wertezerfall nicht ohne Folgen für Hans Demokratieverständnis bleiben. So widerspricht er in seinem Essay über die Digitale Rationalität der These von Eli Pariser, dass das Internet den öffentlichen Raum der klassischen Medien zerstöre, der für einen demokratischen Diskurs notwendig sei, da es das Private veröffentlicht und jeder seine eigene Egozentrik einbringt. Während in den klassischen Medien als einer demokratischen Öffentlichkeit die politischen Themen abgewogen diskutiert werden und die Teilnehmer dabei zu einem Ergebnis gelangen, das dem Gemeinwesen dient, zerfällt die demokratische Gemeinschaft, wenn jeder seine privaten Vorlieben im Internet publik machen kann. Diese Personalisierung entmündigt gar und zerstört mit der Öffentlichkeit die Demokratie.

Han bezweifelt indes, dass die klassischen Medien überhaupt die Bürger gut informieren. Ja, womöglich ist der Netz-Bürger sogar besser informiert als der Bürger der alten Öffentlichkeit. Anstatt einer egozentrischen Personalisierung den Weg zu ebnen, führt das Internet für Han zu einer Dezentrierung, die ihrerseits indes ähnliche zentrifugale Kräfte entfaltet, viele Stimmen intoniert, die aber ebenfalls zu keiner gemeinsamen Melodie mehr gelangen. Han konstatiert: »Aber aus diesen Egos bildet sich kein Wir zum kommunikativen Handeln. Zur Entpolitisierung der Gesellschaft führen also der Zerfall der kommunikativen Öffentlichkeit und die zunehmende Narzissifizierung des Selbst.« (10)

Dieses Wir-Gefühl ist nach Han für eine demokratische Politik schlicht notwendig. Daraus entsteht eine politische Masse, die einer politischen Idee folgt. Im digitalen Schwarm geben die Bürger dagegen ihr Profil und ihre Kontur nicht auf, bleiben sie isolierte Egozentriker, vertreten sie partikuläre Interessen. Das verhindert, dass sie eine gemeinsame politische Gestalt annehmen, gehen sie nicht in der Masse auf, beten sie also bestimmte Freund-Feind-Unterscheidungen nicht mehr nach. Daher kann es denn auch in einer Schwarmdemokratie als mögliche Perspektive der Internet-Welt keine Ideologien und keine Parteien geben, keine Massen und keine Macht, stattdessen nur Fragmentierung und Atomisierung. Denn die Bürger ordnen sich keiner gemeinsamen Idee unter, die das Allgemeinwohl bestimmt. Es verwundert nicht, dass Han Star einer Szene konservativer Kulturkritik ist, in der man glaubt, die richtige Idee vom Allgemeinwohl $\mathrm{zu}$ vertreten und man nicht sehen will, dass jede Ideologie genauso berechtigt ein anderes Allgemeinwohl propagieren kann.

Statt Parteiendemokratie herrscht jetzt für Han ein digitaler Materialismus, den Rousseau erfunden haben soll - eine mindestens amüsante Rousseau-Interpretation, die diesem höchstens partiell gerecht wird. Rousseaus Allgemeinwille entsteht nicht durch Kommunikation der Bürger, sondern durch schlichte Überlegung, genauer Berechnung, was das allgemeine Gute für ein Gemeinwesen ist. So kommen denn auch die Gesetze ohne Diskussion der Bürger zustande, ja es darf gar nicht kommuniziert werden. Aufgeklärte Bürger trennen nach Rousseau nur kleine Differenzen. Ließe man diese beiseite, dann ergibt das den Allgemeinwillen. 
In diesem Sinn interpretiert Han eine Echtzeitdemokratie im Netz. Anstatt langer Diskurse wird in Echtzeit getwittert, lassen sich daraus unmittelbare Entscheidung ableiten, so dass Han von einer Präsenz-Demokratie spricht, die der einzelne in seiner Freizeit vom Bildschirm aus betreibt. Dazu reichen Mausklicks, bedarf es keines langwierigen herrschaftsfreien Diskurses. Die Öffentlichkeit rutscht dabei in die Privatheit, ist Muße und nicht mehr Arbeit. Die Distanz schaffende Repräsentation wird von der Präsenz verdrängt. Ständig könnten Wahlen in den sozialen Medien stattfinden. Implizit lautet Hans Kritik, dass sich in der Schwarm-Demokratie die Bürger nicht mehr von den weisen Eliten lenken lassen, sondern selber entscheiden. Dieser Einwand lässt sich aber nur vorbringen, wenn man voraussetzt, dass die sogenannten Eliten wirklich weise sind.

Die Transparenzgesellschaft entpolitisiert jedenfalls die Politik. Das führt gerade nicht zur Befriedung von sozialen Konflikten, sondern verschärft den Kapitalismus, weil der ausgeleuchtete Egozentriker besser ausgebeutet werden kann. Denn es herrscht der Kampf um das Überleben, um das reine Leben, der nur noch vom Leistungsimperativ geprägt wird. Das führt zur Hyperaktivität und meidet jegliche Verantwortung. Der ethische Imperativ >Du sollst! ist nach Han vergleichsweise harmlos gegenüber dem Imperativ $>$ Du kannst!

Der bloß noch arbeitende Mensch wird depressiv. Er verfällt einer isolierenden Müdigkeit und vereinsamenden Erschöpfung. Nach Han würde Hannah Arendt dem arbeitenden Menschen den handelnden entgegenstellen, was entweder in das Arbeiten zurückfiele oder in die Kontemplation führt, der Arendt auch zuneigt. Nur hat Han Arendt damit gründlich missverstanden und nicht begriffen, dass Handeln für Arendt Kommunizieren heißt, was ja in der Internetwelt angeblich gar nicht mehr stattfindet. Doch man darf bezweifeln, dass Arendt dem zustimmen würde, war sie doch weder pessimistisch noch konservativ.

Doch Han sucht auch nach Auswegen. So setzt er in seinem Essay Müdigkeitsgesellschaft der Erschöpfungsmüdigkeit eine inspirierende Müdigkeit entgegen: »Die Erschöpfungsmüdigkeit ist eine Müdigkeit der positiven Potenz. Sie macht unfähig, etwas zu tun. Die Müdigkeit, die inspiriert, ist eine Müdigkeit der negativen Potenz, nämlich des nicht-zu. Auch der Sabbat, der ursprünglich aufhören bedeutet, ist ein Tag des nicht$\mathrm{zu}$, ein Tag der befreit ist von jedem um-zu, um mit Heidegger zu sprechen von jeder Sorge. Es handelt sich um eine Zwischenzeit. (...) Es ist der Tag der Müdigkeit. Die Zwischenzeit ist eine Zeit ohne Arbeit, eine Spielzeit«. (62)

Dass eine solche Gesellschaft, eine Müdigkeitsgesellschaft kommen soll, das ist ByungChul Hans Hoffnung. Aber woher sie kommen soll, das sagt er nicht. Womöglich an Pfingsten, das er mit solcher Müdigkeit verbindet! Wenn aber die Leute daran nicht mehr glauben? Am Anfang von Agonie des Eros hofft er in der Hölle des Gleichen auf jenen absolut anderen, der eine Apokalypse auslöst. Beruhigend bleibt, dass Apokalyptiker mit ihrer Drohung nur die Zeitgenossen zur Umkehr bewegen wollen. Dann kann man neugierig zuschauen, wie weit er kommt. 